\title{
The Philosophy of the Cosmos as the New Universal Philosophical Teaching about Being
}

\author{
Oleg Bazaluk \\ Doctor of Philosophical Sciences, Professor, Pereiaslav-Khmelnytskyi Hryhorii Skovoroda \\ State Pedagogical University (Kyiv, Ukraine) \\ E-mail: bazaluk@ukr.net \\ ORCID: 0000-0002-1623-419X
}

\section{Larysa Kharchenko}

$\mathrm{PhD}$, Associate Professor, Pereiaslav-Khmelnytskyi Hryhorii Skovoroda State

Pedagogical University (Pereiaslav-Khmelnytskyi, Ukraine))

E-mail: harchenko_lora@ukr.net

ORCID: 0000-0002-5731-0531

\begin{abstract}
The authors' understanding of neuro-programming is the result of the impact on the human brain of information and communication technology (including educational one), through which in the human brain the programs of manifestation in the ontogenesis of internal creative potentials are written. This article summarizes the history of the formation of key neuro-programming technologies of the human brain as well as proves that the changes in the society's worldview are caused by the possibilities and quality of neuro-programming technologies that society uses. Having influence over worldview stereotypes and behaviour set by the society, neuro-programming technologies essentially ensure the national security of any state and the peaceful coexistence of states in the regions and on the planet as a whole. Using historical and philosophical methods, methods of conceptualization, systematization, modeling, etc., the authors have come to the conclusion that the modern world lies in a confrontation of security strategies, in which neuro-programming technologies play a key role.

Keywords: technologies of neuro-programming, educational technologies, Plato, national security, human brain, the state
\end{abstract}

Received: February 25, 2018; accepted: May 5, 2018

Philosophy and Cosmology, Volume 21, 2018: 6-13

DOI: $10.29202 /$ phil-cosm/21/1

(C) Bazaluk, Oleg, 2018

(C) Kharchenko, Larysa, 2018 


\section{Introduction}

In the theory "Evolving Matter," Oleg Bazaluk postulated two basic provisions: ${ }^{1}$

1. Evolution is complication of the structure of matter, the types of interaction and the environments, though in the unity and struggle of opposites. Speaking about evolution as complication of the Universe, the author means complication of three components of physical reality: 1) the structure of matter; 2) the types of interaction (relations) between the structures of matter; 3) the environments, in which complication of these structures and interactions are carried out, and which, to a varying degree, determine environmental characteristics [Bazaluk, 2016: 35].

2. The complication of any state of matter is based on three factors and two causes of evolution. To the factors of evolution as the complication, the author assigns:

a) Continuity of self-complication of the structure, the types of interaction and the environments of any state of matter, supplemented by blocks of continuous selfcomplication and the principle of dominance of continuous block self-complication.

b) Nonlinear complication of the structure, the types of interaction and the environments of any state of matter, which is added by the factors: hierarchical nonlinear complication and direction of nonlinear hierarchical complication.

c) Isolation of complication (or, Plotinus' setup for "self-assembly"). ${ }^{2}$

Oleg Bazaluk attributes the causes of evolution as complication to [Bazaluk, 2016: 136]:

a) Active principle, which is inherently the basis for the initial elements of any state of matter and forms self-complication.

b) Natural selection as the impact of the external environment.

Interaction of the active internal principle of any state of matter with natural selection as the impact of the external environment forms a regulatory compromise [Bazaluk, 2016: 136].

On the basis of the proposed postulates, Bazaluk systematized the accumulated knowledge about the evolution of the Universe, biological life and human and came to the conclusions [Bazaluk, 2016: 129-130]:

1. Complications of the structure of matter, the types of interaction and the environments in our Universe have been carried out by hotbeds, continuously and nonlinearly, over proximately 13.7 billion years. Complication of the structure and functions of the Universe happens under the influence of the same (Universal) factors and causes of evolution. Thus, in the course of evolution, complication of the very factors and causes of evolution themselves happened, which led to the formation and development of the $\mathrm{n}^{\text {th }}$ number of states of matter.

2. Each state of matter is a new level of complication of the structure of matter, the types of interaction and the environments. In consequence of the complication of each new state of matter comes the formation of invariant hierarchies, providing fixation of the state of matter in the structure of the Universe and its co-evolution with other states of matter. Each new state of matter brings new opportunities for the organization of the circulation of substances, energy and information, as well as ways of moving in space.

3. Studying the modern scientific and philosophical theories of evolution, the author discovered the unity of micro and macroevolutionary processes. The basis of this unity is the variability of the factors and causes of evolution. The transition from the Microcosm to the Macrocosm in Inert Matter, Living Matter, Intelligent Matter and any other states of

${ }^{1}$ We shall consider only those provisions of the theory "Evolving matter," which are necessary for building the theory of education. The arguments of these provisions, as well as the theory of evolution itself, can be found in the book [Bazaluk, 2016].

${ }^{2}$ See [Plotinus, 1952]. 
matter is a consequence of the impact of regulatory compromise and variability of the factors and causes of evolution, which are complicated during time, complicating the structure and functions of the Universe.

4. During the work on the evolution models of three states of matter that are known to modern science: Inert Matter, Living Matter and Intelligent Matter, the author discovered and considered the so-called "transition" states of matter. The author defined them by the terms "Bioinert" and "BioIntelligent" matter [Bazaluk, 2016]. Namely, the "transition" states of matter complicate the systematization of knowledge, because, on the one hand, they are structurally and functionally the highest structures of the "mother" state of matter, on the other hand, they are included in the more perfect blocks of the "daughter" state of matter. "Transition" states of matter are the maximum possible limit of continuous and nonlinear complication of the "mother" state of matter. Modern science does not know of any examples when in the "mother" state of matter, complication of the structure, the types of interactions and the environments went beyond the hierarchy of a transition state of matter.

5. On the example of the Solar System, only one sequence of complication of the structure of the Universe can be seen: Inert Matter $\rightarrow$ Living Matter $\rightarrow$ Intelligent Matter, or alternatively, taking into account "transition" states of matter: Inert Matter $\rightarrow$ Bioinert Matter $\rightarrow$ Living Matter $\rightarrow$ BioIntelligent Matter $\rightarrow$ Intelligent Matter. The modern understanding of evolution and co-evolution of these states of matter is considered in the standard model of the Universe, the synthetic theory of evolution and the concepts of noogenesis.

6. At the scale of the Solar System, the states of matter have been formed sequentially, at intervals of approximately 3 billion years:

a) Approximately 6(5.5) billion years ago, in the Milky Way Galaxy, the Solar System was formed - one more hotbed in uneven, continuous and nonlinear block complication of the Universe. Vladimir Vernadsky denoted the richness of the structure and the manifest functions of our Universe by the term of "system of Inert Matter."

b) Approximately 3.5 billion years ago, as a result of geological evolution, on the Earth, the first biological organisms emerged and gained a foothold. Over 3 billion years they formed the system of Living Matter, which in science was denoted by the term "biosphere".

c) Approximately 6-7 million years ago, as a result of neuroevolution, on the basis of the highly developed biosphere of Earth, the first structures of Intelligent Matter emerged. This started the formation of the noosphere.

Extrapolating the results of studies of the Solar System on the structure of the Universe, Oleg Bazaluk came to a whole series of conclusions. Let us consider the following [Bazaluk, 2016]:

At the scale of the Universe, five states of matter are evolving as a minimum. One of the unknown states of matter to modern science is the "mother" state for our own Universe. The Universe is developing within it, and probably separate features, which are ascribed by humanity to the Universe (e.g. gravitation) are actually manifestations of states of Matter Y. The second unidentified state of matter by humanity emerged on the basis of highly developed Intelligent Matter. In the model, it is indicated as the state $\mathrm{X}_{1}$. The model admits the state $\mathrm{X}_{2}$, from which is probably formed the state $\mathrm{Y}$ and the so-called "new Universe" emerged. These successive stages of complication of the states of matter can include an $\mathrm{n}^{\text {th }}$ number of chain links, but the result is the birth of a "new Universe" when saving an "old Universe".

${ }^{3}$ To be precise, Vernadsky used the concept "Inert Substance." In the works, we gave arguments for the advisability of replacing the term "Inert Substance" with "Inert Matter" [Bazaluk, 2016]. 
1. The complication is happening uneven and through "hotbeds." If we suppose that the Universe perceived by humanity is a fragment of space in which continuous and nonlinear complication of the structure of matter, the types of interaction and the environments are happening, then it is possible that "Our Universe" is nothing other than a plurality of Universes which are observed by us in one or other state of complication.

2. The earth's noosphere in the modern state is only the beginning of noogenesis. In future, a minimum of 3 billion years of continuous and nonlinear complication are to be expected. Humanity will expect a lot of changes. For the scale of these changes, we can judge from the example of the complication of Inert Matter and Living Matter at the scale of the Solar System. Inert Matter had been complicated for 3 billion years from quarks to polymers, Living Matter from RNA molecules to mammals. The complication of the Earth's Intelligent Matter began with neural ensembles of subconsciousness that separated out the family of the most progressive primates (hominid) from the animal world. For 6-7 million years neuroevolution, sociocultural evolution and the evolution of the technologies of the most ancient hominids have brought us to the exploration near-Earth space. Taking into account that in 1 billion years the condition of Earth will be unsuitable for biological life due to physical changes in the sun, a strategy of the evolution of Intelligent Matter becomes clearer: a) as much as possible, to obtain full liberation from factors of the biological environment; substituting them with more reliable and controlled artificial constructs; b) the exploration near and far space; c) the complication of the sociocultural environment and technologies to a level, which provides the noosphere relief from the destructive influence of the cosmos.

The theory "Evolving matter" allows the possibility of a simultaneous presence of a certain number of states of matter in the Universe, at different stages of their complication. We can speak of the five states of matter with the greatest probability:

1. Matter $Y$ is substance, the types of interaction and the environments, which preceded Inert Matter, and at the scale of which Inert Matter arose and continues to become more complicated. Matter $\mathrm{Y}$ is not recognized and is not studied by modern science.

2. Inert Matter is ours and other Universes. At present, the Standard Cosmological Model gives the most complete understanding of continuous and nonlinear complication of Inert Matter.

3. Living matter is the biosphere, which naturally arises in various parts of the expanding Universes, on space objects with a certain range of physicochemical characteristics. The synthetic theory of evolution provides the most complete understanding of the continuous and nonlinear complication of the biosphere in individual cosmic object.

4. Intelligent Matter is noospheres, which naturally arise on the basis of highly developed biospheres. Unlike Living Matter, Intelligent Matter is neither structurally nor functionally connected to cosmic objects. In modern science, the cosmic significance of Intelligent Matter is not recognized. The theory that explains the evolution of Intelligent Matter does not exist.

5. Matter $X_{1}$ is a state of matter which naturally arise in highly developed noospheres. Modern science does not recognize and research this state of matter.

The states of matter listed above are formed sequentially, through "hotbeds," with a certain range of physicochemical characteristics, and at intervals of approximately 3 billion years. The totality of states of matter forms continuous and nonlinear complication, which is 
denoted by the term "Universe" in modern science. The theory "Evolving matter" allows the existence of a certain set of Universes and, accordingly, cosmic biospheres, noospheres, etc.

\section{The way to achieve philosophical knowledge}

The fundamental difference between the philosophers' desire for wisdom and the scientists for erudition is hidden in their relation to knowledge. Modern knowledge of cosmologists, biologists, anthropologists or scientists from any other scientific disciplines, Plato would compare the specialized knowledge of tradesmen, which were valuable only in their branch of knowledge but were less useful in the others. ${ }^{4}$ The undervaluing of philosophy and philosophical knowledge has led to the fact that modern research in cosmology, biology and neuroscience is completely unrelated and does not enrich each other through explorations.

In the philosophical tradition, knowledge is the way to the Divine, or, in the terminology of Modern philosophy, it is the way to the ontology of the cosmos. Philosophical knowledge is a universal culture that goes back to the aristocratic ideal of early Greece, which can only be understood in the context of the history of Antiquity. The recognized authorities in this field research Werner Jaeger and Pierre Hadot proved that the stages of the development of philosophical knowledge, in essence, determined the stages of the development of ancient culture. ${ }^{5}$ Philosophical knowledge was the basis on which the Greeks built a holistic understanding of the world, embracing the unity of the ontology of human being, the state, and the cosmos. ${ }^{6}$

The way to achieve philosophical knowledge lies in "the search of wisdom." If we take into account that in Plato's view only God was wise, then "the search of wisdom" gave the possibility for philosophers to cognize the ontology of the cosmos through the synthesis of scientific knowledge in the universal (all-encompassing) philosophical understanding of the world. The teachings of Aristotle, Plotinus, Porphyry, and others enriched Plato's ideas about knowledge. For example, Aristotle defined scientific knowledge as "second philosophy" in philosophical knowledge as "first philosophy" and emphasized its crucial importance in the formation of philosophy as universal science. In Aristotle's view, philosophy as a way of life allowed a philosopher to concentrate on a more detailed study of nature, or in modern terminology, on applied and fundamental scientific research. ${ }^{8}$ Only in the $19^{\text {th }}$ century, William Whewell, a theologian and philosopher, coined the term "scientist," by which he designated philosophers specializing in the detailed study of some phenomena of nature, including physical, mathematical and social realms.

If we follow the philosophical tradition, then the modern, completely new and impressive level of scientific argumentation, which was accumulated in numerous branches of knowledge, cannot a priori deny philosophy. On the contrary, it should be united through a universal philosophical theory and brought the modern culture to new levels of perfection.

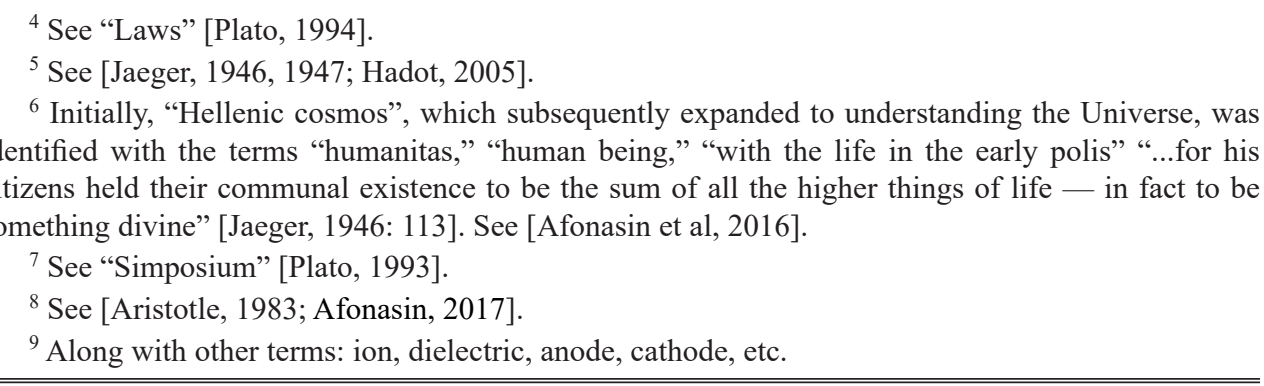




\section{The new universal philosophical teaching about being}

The theory "Evolving matter" is the scientific and philosophical theory that in a certain way systematizes the scientific knowledge of the last four centuries and enriches the ontology of the cosmos with new meanings. It gives the possibility of creating the philosophy of the Cosmos, that is a new universal philosophical teaching about being, which unites the disparate scientific knowledge of cosmology, biology and neuroscience through the general philosophical idea "Those Who Transform the Universe." It follows from the theory "Evolving matter" that each state of matter is a part of the whole, which, by its appearance and complication, causes the complication of the whole. The cosmos in the philosophical understanding is the absolute One ${ }^{10}$ that is much wider than the scientific understanding of the cosmos as the Universe. In the cosmos as the One, the idea "Those Who Transform the Universe" is already present as the cause of all that is happening in the world. The cosmos as the One is the absolute unity of the human ontology (Intelligent Matter) and any other state of matter in the ontology of the cosmos. It is not in the meaning of unification, but in the meaning of the common initial principle, the primogeniture. In fact, Plotinus' understanding of the One, which in the Middle Ages was transformed into the understanding of the Triune God, is the principle of being, that is what was, is and will be in the Universe. ${ }^{11}$

The philosophy of the cosmos, as the modern philosophical teaching about the being of Dasein-the One, allows us to consider the Universe not as a narrowly specialized cosmological model, the Lambda-CDM model, but as the synthesis of knowledge in cosmology, biology and neurobiology through common fundamental philosophical ideas. In the philosophy of the cosmos, the Universe is continuously and nonlinearly complicating order inside and between a certain numbers of states of matter. Each state of matter has a certain degree of freedom, which allows to appear and become fixed in the system of the "mother" state of matter. The key meanings of any state of matter are in the term Dasein. Dasein of any state of matter is a certain way of presence in being. This is adjoint presence to oneself, to the complication of one's structure, functions and manifestations, as well as to "mother" and other states of matter. It is a concentration of the experience of being of the previous states of matter in the newly emerged state of matter. The complication of the being of Dasein of each new state of matter alters the existing order in the being of Dasein-the One. Martin Heidegger revealed to a certain extent the meanings of the being of Dasein of Intelligent Matter. However, these meanings are even deeper, because man is present not only in himself and for himself. The complication of man, like a certain way of being, occurs in the complication of the being of the "mother" state of matter and leads to nonlinear complication of the entire order of Dasein of previous states of matter in our Universe. The being of Dasein's Man concentrates in itself the experience of being Dasein of Living, Inert and Y-matter, which gives it the power of cosmic one and transforms it into the potential of the being of Dasein-the One.

The Dasein of "daughter" state of matter is a new level of complication of the structure of matter, the types of interactions and the environments, which gives new possibilities for continuous and nonlinear block complication of the Universe. This is a new way of life: being-there for oneself, that is for the complication of the Earth's noosphere and, at the same time, being-there for others, that is for the complication of the Earth's biosphere and the Universe. To become part of the "mother" state of matter and eventually to create its own system in it means to make certain changes in the complication of the "mother" system and,

${ }^{10}$ We use Plotinus' terminology that contains the important meanings of the teachings of Plato and Aristotle for our research [Plotinus, 1952].

${ }^{11}$ See [Plotinus, 1952]. 
consequently, in the complication of the Universe. Each new state of matter transforms the Universe by the fact of its appearance and fixation, i.e. its Dasein.

The number of appearance of new states of matter in the "mother" system does not correspond to the number of fixed states of matter. To become part and to create a system in the "mother" state of matter, each new state of matter should possess two basic qualities:

1. The possibilities to make full use of the material, energy and information resources of the "mother" state of matter.

2. The possibilities to transform the "mother" system in order to create conditions for continuous and nonlinear block complication of its own structure and functions, i.e. to create the "daughter" system.

It follows from the theory "Evolving matter" that each new state of matter, after about 2 billion years of continuous and nonlinear block complication, reaches a certain perfection in the organization of its system, and turns into the "mother" system. That is, the "daughter" state of matter reaches such a level of internal complication that allows the appearance of new qualitative structures and functions in the system, which can overcome the boundaries of the "mother" system to become part in being of Dasein-the One and to create its own being of Dasein in it. We called these structures "transitional" states of matter. From a certain set of continuously and nonlinearly emerging transitional states of matter, in the "mother" system only one state of matter is fixed, whose structure and functions use the resources of the "mother" system most effectively to create its own being of Dasein.

The "daughter" system occupies an "insignificant" place at the scale of the "mother" system. However, despite the fact that the scale of the presence of each new state of matter in the already existing hierarchy of the Universe is significantly reduced, its ability to transform the Universe, on the contrary, is increasing. The reason lies in the being of Dasein of a new state of matter, in which the experience of the being of the previous states of matter is concentrated. In the being of Dasein of each new state of matter, the Dasein-the One finds new ways of presence for itself and taking care of its being. For the being of Dasein-the One, the being of Dasein-the Universe, as well as the being of Dasein of any other state of matter, is the necessity of being, that is, to embody the power of its Dasein in continuously and nonlinearly complicating ways of presence. Therefore, each new state of matter, as a new manifestation of Dasein-the One, not only complicates the structure, functions and manifestations of the already existing order of the previous states of matter, as the earlier manifestations of Dasein-the One but also concentrates the experience of being of their Dasein in itself. Owing to the concentration of the experience of being in the being of Dasein of a new state of matter, Dasein-the One preserves the possibility of taking care of its being and ensuring the unity of the varieties of its manifestations. ${ }^{12}$

\section{Conclusion}

The theory "Evolving matter" gave the possibility of creating the philosophy of the cosmos, which is a new universal philosophical teaching about being. The new teaching united the disparate scientific knowledge of cosmology, biology and neuroscience through the general philosophical idea "Those Who Transform the Universe." In the philosophy of cosmos, the Universe is regarded as an absolute unity of the ontology of Intelligent Matter and any other state of matter in the ontology of the cosmos, or as the being of Dasein-the One. The being of Dasein-the One finds new ways of presence for itself and taking care of its presence in the being of Dasein of each new state of matter. For the being of Dasein-the One, the being

${ }^{12}$ See [Bazaluk, 2017]. 
of Dasein-the Universe, as well as the being of Dasein of any other state of matter, is the necessity of being, that is, to embody the power of its Dasein in continuously and nonlinearly complicating ways of presence. Therefore, each new state of matter, as a new manifestation of Dasein-the One, not only complicates the structure, functions and manifestations of the already existing order of the previous states of matter, as the earlier manifestations of Daseinthe One but also concentrates the experience of being of their Dasein in itself.

\section{[Ld] References}

Afonasin, Eugene. The 'relics' of the past. Aristotle — the historian of philosophy. $\Sigma X O \Lambda H$ (Schole). Vol. 11.2. 2017: 570-607.

Afonasin, Eugene, Anna Afonasina, and Andrey Schetnikov. Ancient Cosmos. Essays on Astronomy and Cosmology in Antiquity. St. Petersburg: RHGA, 2016.

Aristotle. Collected Works in Four Volumes. Moscow: Mysl', 1976-1983.

Bazaluk, Oleg. The Theory of Evolution: From a Space Vacuum to Neural Ensembles and Moving Forward. Cambridge Scholars Publishing, 2016.

Bazaluk, Oleg. The Theory of Education: "Those Who Transform the Universe" (New Book Announcement). Future Human Image, Volume 8, 2017: 9-13.

Jaeger, Werner. Paideia: The Ideals of Greek Culture: Volume II: In Search of the Divine Centre. Basil Blackwell, Oxford, 1947. https://archive.org/stream/werner-jaegerpaideia-the-ideals-of-greek-culture-vol2\#page/n1/mode/2up.

Hadot, Pierre. Philosophy as a Way of Life: Conversations with Jeannie Carlier and Arnold I. Davidson. Trans. V. A. Vorobyov. Moscow, St.-Petersburg: Stepnoy veter; Kolo Publ, 2005.

Klepko, Serhiy. Pedagogy of Peace and Philosophy of War: the Search for Truth. Future Human Image, Volume 7, 2014: 46-49.

Plato. Collected Works in Four Volumes. Moscow: Mysl', 1990-1994.

Plotinus. The Six Enneads. Translated by Stephen MacKenna and B. S. Page. Chicago: Encyclopaedia Britannica, 1952. http://www.sacred-texts.com/cla/plotenn/index.htm. 